Reporting Date: October 197? Issued: December 1975

\title{
Description of Collisionless Plasmas by Classical Field Equations
}

by

\author{
Gary S. Fraloy
}


Pristed in the United Statet of Americe. Available from Nutional Technical Informiation Service

I.S. Department of Cnmmerce

5285 Port Rnyal Rused

Springfield. VA 22151

Price: Printed Copy $\$ 4.50$ Micrifiche $\$ 2.25$

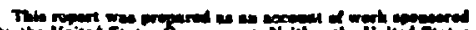

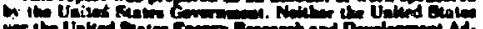

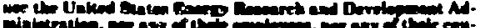

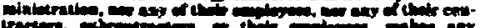

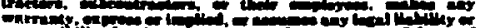

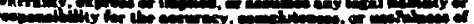

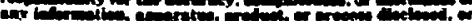

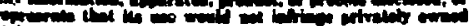
tint. 
ClassICAL FIEL ENHTIONS

by

Gitry S. Praiky

\section{MBSTRACT}

Classical fleld equatlons are derived from quaneus fielde to oteatin a dfferent and posslbily sleppler description of a collisionless plinspo. The methoul $1 \mathrm{~s}$ to cake the simulenneous linte, $h, \ldots, 0$, of citsiged

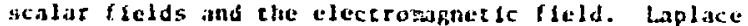
transforms: for perturbations in a unliorp relativist de blasma are cospazed uth correspondlak result tron the Maxwe11-Vlasov equations. For the nonlinear case, a distribution function defined on the classlcal flelds its shown to satdsty the vlasov equetit ton.

\section{DERIVATION}

the are interested in inding a different description of a collisionless plasan. In particutar, one wh about the sathe physteal conetene as the stindard desertpeton bue which any, in soze cises, have a stmpler mathenteleal seructure, it is useful to eximine a derivation of the standard description the Vlasov equat lon. An ensemble averabe over classical lonized parteles gives the bicke hierarchy of equntions. " when second order correlations are neglected, the first equat lon of the hiferarchy reduces to the jlasov equation. It 1 s penerally assuned that in the 1 indt $e, m \rightarrow 0$, bach $\mathrm{c} / \mathrm{m}$ and "e remalning constant, where ne" and in are che particle densiey, eharge, and mass. resjectively, second order cotrelations disappear. We maly then wrice

$$
s_{c p}=\lim _{e+m+0} \sum s_{c}
$$

wher: $S_{c}$ represents che equations for classical particles, $S_{c p}$ the equationb for a collizionless plasma, and $\Sigma$ an ensemble average. Because the

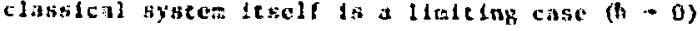
of a quantum syster $s_{Q}$.

$$
s_{c p}=\lim _{c, m \rightarrow 0} \sum \lim _{h \rightarrow 0} s_{q} .
$$

Here we Invest figte the case whert sone of the ifwits are exchanged. The lnitlad delt is

$$
S_{c p}=\frac{11 n}{c, p+h \rightarrow 0} S_{Q} .
$$

where the raclos of $c, m$, and h remin constant. other derliptlons of plasen phenonena fros quaneus systeas ${ }^{2-4}$ have corresponded to the 1 inle $h+0$. Because these 11 mita are bingular, cheir exclunge may not give the same resulis. The resulcs muse be Investigated in ersch case. He neglect spin effects: $s$ a ts represenced by charged scalar flelds $\left(\phi_{q}\right)$ for each charge spectes (q) and the electromagnetic fleld $(A)$. The equations are 


$$
\begin{aligned}
& a_{3} A^{v}=4 r \sum_{q}
\end{aligned}
$$

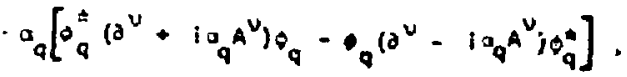

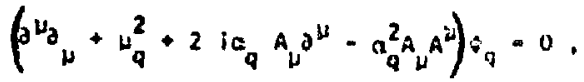

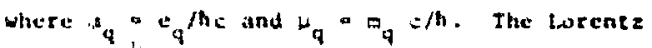
gauge $a A^{\text {l. }} 0$ dis used. He use caussian unics. the fledds are quantized by conentation rules such as

$$
\begin{aligned}
& {\left[a_{0} \theta_{Q}(\vec{x}, t), \theta_{Q}(\vec{y}, t)\right]-i c h \delta(\vec{x}-\vec{y})} \\
& {\left[a_{0} A_{\mu}(\vec{x}, t), A_{y}(\vec{y}, t)\right]=-i 4 a c h \delta\left(\vec{x}-\vec{y} g_{\mu \nu}\right.}
\end{aligned}
$$

where $9_{00}=1--9_{11}=-9_{22}=-9_{33}$.

The fleits diffor iras cheli scandard repre-

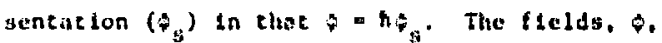
have the same dimentonality as the clectemagnete cleld; the square of thelt derlvatives gives an encrey densicy.

The fleld equatioas are Invariane under the 11ate; the tonzutators Ulsappear. We may then incerptet che flelds "classfeally" as complex nubber funcelons. The ney syece 13 deteralnistic although it has fince da broglie waveiangehs. We are Interesced in the case where the de Brogle vavelengch is sanll compared to any ocher scale length. Le my be considered an Infinfeceland; Its prectse volue ts not importane if te is soill enough. It appears teasonable that thase equations correspond to a collasionless plasmil. The colldsionless approximation broulhs over partcle effeces. Converaly, the quartization adds partfcle-like of discrete of focts to the fleldr.

In the absente of an electromagnet fe flald, lise charge dengley due to poslesve energy plane

$$
\begin{aligned}
& 0(\vec{i}, t)=0 \sum_{i} \exp |1 \vec{i}, \vec{x}| \\
& -\sum_{k} a_{k}^{a} k-i u_{k-i} \exp \left(1\left(u_{k-2}-u_{k}\right) t\right)+z . c .
\end{aligned}
$$

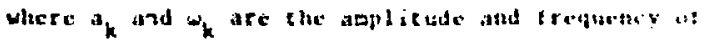
the plane vave with wave numier $h$. For a tallote

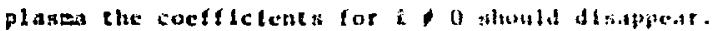
Thls will be crue over an enseeble averate it the

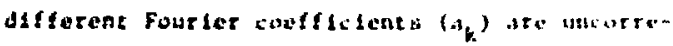
lated. In an Indivldual plates. the number at teras in the sum over $h=0\left(1 / h^{3}\right)$. Then, tor uncorrelated $a_{k}$, the denaley corteletene fut i 0 lo $0\left(\mathrm{~h}^{3 / 2}\right)$. In a second lioic (h- $0.1 .4 \ldots$,

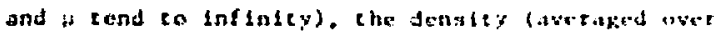

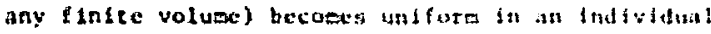
planga for uncorrelated $a_{k}$. In veseral, if the plasea changes over a scale length $b_{1}$, there sluwbl be correlations between wave nughers tor

$$
|A k|=o\left(1 / k_{1}\right)
$$

21. CONARISOH WITH THE MUAELL-VIASOF ENUATIOSS A. Linear Percurbations

the vill compare solut tons tor the lute $1: 1$ value problew in a untorm relativitic planda with $n=$ zero order electrumatrecte t1eld. Longleudial and cransverse waves are dons smarafoly vith the coupling between the linnored. The: charged paretele flelts are separaced taco zere order and flrat order pares. Calculat lons for each charged particle specios are done sieparately.

$$
\begin{aligned}
& \theta_{0}+\theta_{1} \\
& \phi_{0}=\sum_{k} s_{k} \exp \left[1\left(\vec{k} \cdot \vec{x}-\cdots_{k}\right)\right] . \\
& \phi_{1}=\sum_{k} b_{k}(c) \exp [1 \vec{k} \cdot \vec{x}],
\end{aligned}
$$


where $w_{k}>0$. For longltudinal waves, $A$ has a teslike pare

$$
A^{0}-\sum_{k} A_{k}^{0}(t) \exp (2 \vec{k} \cdot \vec{x})
$$

ano a space-11ke part

$$
\Lambda^{*} \cdot \sum_{k} e_{k}^{a} a_{k}(t) \exp \mid(\vec{k} \cdot \vec{x} \mid, \cdots-1,2,3
$$

wher of 10 ande vector porallet to $k$. The Lorentz gauge glva:

$$
-1 k h_{k}(t)-\frac{\partial}{d t} A_{k}^{0}(t)
$$

Foz transverse waves theze 10 only a space-like part with each polarize ion perpendlcular cok. The llnear1red equaclone ore

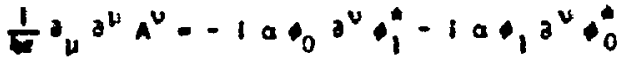

$$
\begin{aligned}
& -d^{2} A^{v} \bullet_{0}+c . c . \\
& \left(\omega_{a}+u^{2}\right) v_{1}+2+a a^{\nu} \theta_{0}=0 .
\end{aligned}
$$

For longitudinal case the Laplice sraneform of Eq. (10) gives

$$
\begin{aligned}
& \left(\frac{s^{2}}{c^{2}}+k^{2}+u^{2}\right) b_{k}(s)=\frac{1}{c^{2}}\left(s b_{k 0}+b_{k 0}\right) \\
& -21 \frac{0}{c} \sum_{l} \lambda_{2}^{0}\left(s+1 \omega_{k-2}\right) \\
& \cdot v_{k-2}\left[2-\frac{\vec{k} \cdot \vec{L}}{\vec{k} \cdot \vec{L}}\left(s+\mid \omega_{k-1}\right)\right]
\end{aligned}
$$

$$
-21 \frac{a}{c} \sum_{l} A_{w 0} a_{k-l}\left(\frac{\vec{k} \cdot \vec{l}}{\vec{k} \cdot \vec{l}}-1\right) \text {, }
$$

where

$$
b_{k C} \equiv b_{k}(t-0) \cdot b_{k 0}^{\prime} \equiv \frac{b_{k}}{d t}(t-0) .
$$

$$
A_{10} \equiv A_{2}^{0}(t-0), A_{20}^{\prime} \equiv \frac{\partial A_{2}^{0}}{\partial t}(t-0) .
$$

The craniforn of Eq. (9) for $v=018$

$$
\begin{aligned}
& \frac{1}{a}\left(\frac{s^{2}}{c^{2}}+k^{2}\right) A_{k}^{0}(s)=\frac{1}{4 \pi c^{2}}\left(s A_{k 0}+A_{k 0}^{\prime}\right) \\
& +\frac{10}{6} \sum_{l} c_{k+2} \\
& -1 \frac{a}{c} \sum_{l} a_{k+2}\left(s+i \omega_{k+l}\right) b_{\ell}^{n}\left(s+i \omega_{k-2}\right) \\
& +\frac{a}{c} \sum_{l} a_{L-k} \omega_{l-k} b_{l}\left(s-i \omega_{l-k}\right) \\
& -a^{2} \sum_{l, j} a_{\ell}^{0}\left(s+i w_{j}-i w_{l, j-k}\right) a_{j} t_{l+j-k}+c . c .
\end{aligned}
$$

The subaticution of Rq. (11) Into Eq. (12) couples che fourler wodea of $A^{0}$. The coupling between $A_{k}^{0}$ and $\Lambda_{l}^{0}$ is of tie cype $a_{j}^{*}+t^{*} k^{*}$ For a ualform plasen the coupling $=0(\mathrm{~h} 372$ ) since the different of are incoherent. The coupling disappears in the lint $t+0$, and ve have

$$
A_{k}^{0}(s)=s ; 0(k, \theta)
$$

where $S$, chis cource cera, depende on Intelal conditlons, and $D(k, 0)$ Is the Laplace transford "dloperetos zelation." 


$$
\begin{aligned}
& D(k, s)=\left(\frac{s^{2}}{c^{2}}+k^{2}\right)\left\{1+\frac{8 \pi a^{2}}{c^{2}} \sum\left|a_{j}\right|^{2} w_{j}^{2}\right. \\
& x \frac{\left.\left[1-\frac{(\overrightarrow{1} \cdot \vec{k})^{2} c^{2}}{k^{2} w_{j}^{2}}-\left(s^{2}+c^{2} k^{2}\right) / 4 w_{j}^{2}\right]\right)}{\left[w_{j}^{2}(s+1 \vec{k} \cdot \vec{u})^{2} / c^{4}-\left(\frac{g^{2}}{c^{2}}+k^{2}\right)\right]} .
\end{aligned}
$$

where a is the velocity corresponding to $j$. With the substitution

$$
\left|\bullet_{j}\right|^{2} \omega_{j} \rightarrow n_{0} f_{0}(\vec{p}) h c^{2} / 2, \vec{p}=n \vec{j}
$$

where $n_{0}$ is the particle density, $p$ is momentum, and $f_{0} 1 s$ the womentum distribution function.

$$
\begin{aligned}
& \lim _{n \rightarrow 0} D(k, s)=\left(\frac{s^{2}}{c^{2}}+k^{2}\right) \\
& \cdot\left\{+u_{p}^{2} \int \frac{d^{3} p f_{0}(p)\left[1-\left(\frac{\vec{u} \cdot \vec{k}}{c}\right)^{2} / k^{2}\right]}{\gamma(s+i \vec{k}-\vec{u})^{2}}\right\},
\end{aligned}
$$

where $\omega_{p}$ is the plasma frequency.

The square brackets are identical to she equivalent viasov equation dispersion relation ${ }^{1}$

$D_{y}(k, s)-1-1 w_{p}^{2}=\frac{\vec{k} \cdot}{k^{2}} \int d^{3} p\left(\frac{\partial f_{0}(p)}{\partial \vec{p}(s+1 \vec{k} \cdot \vec{u})}\right)$.

Th1s 18 show by integration by parts. The same procedure may be carried out for transverse waves. We obtain

$$
A_{k}(8)=S / D(k, s) \text {, }
$$

where

$$
\begin{aligned}
0(k, s) & =\frac{s^{2}}{c^{2}}+k^{2}+8 \pi a^{2} \sum_{j}\left|a_{j}\right|^{2} \\
& =8 \pi a^{2} \sum_{j}|a j|^{2} \frac{\left(\vec{j} \cdot \vec{e}_{k}\right)^{2}\left(s^{2} c^{2}+k^{2} c^{4}\right)}{w_{j}^{2}(s+i \vec{k} \cdot \vec{u})^{2}} .
\end{aligned}
$$

where $e_{k}$ ls the polarization vector.

$$
\begin{aligned}
& \lim _{h \rightarrow 0} D(k, s)=\frac{s^{2}}{c^{2}}+k^{2}+\frac{\omega_{p}^{2}}{c^{2}} \int \frac{d^{3} p f_{Q}(p)}{Y} \\
& -\left(\frac{s^{2}}{c^{2}}+k^{2}\right) \omega_{p}^{2} \int \frac{\left.d^{3} p f_{j}(p)[\vec{w} / c) \cdot \vec{e}_{k}\right]^{2}}{r(s+i \vec{k} \cdot \vec{u})^{2}}
\end{aligned}
$$

Tr:ansveree waves in a relazivistic plasma have bren investigated by Lerche ${ }^{5}$ and Felderhof, 6 adi.MB others. Apparently, hovever, there is n: explicit expression for the Laplace transfort. comparison can be made oniy for special cases. If the denominator in the last integral is not too small, the last term is a relativiatic correction. and in the nonrelativistic liall we obtaln the standard expresecion

$$
\omega^{2}=k^{2}+\omega_{p}^{2} .
$$

The last tert of Eq. (19), for swall velocitles, parallel to $\vec{k}$, produces the Welbel instabllity.?

\section{B. Monlinear Case}

Here we actempt to find a functlonal of each particle fleld which corresponds to a distribution function, and which satisftes the Maxwell-v1asov equations in s self-consistent way. One difficulty is that a continuous chirge diatritution dues not, In general, satisfy the vlasov equation, e.8.

$$
f(\vec{p}, \vec{x}, t)=g(\vec{x}, t) \delta[\vec{p}-\vec{p}(\vec{x}, t)]
$$


where

$$
\begin{aligned}
\vec{p}(\vec{x}, t) & =\vec{p}(\vec{x}, 0)+[-(\vec{v} \cdot \vec{b}) \vec{p} \\
+ & (\vec{E}+\vec{v} \times \vec{B})] t+o\left(t^{2}\right),
\end{aligned}
$$

and $v$ is the particle velocity. The delta function factor satisfies the Vlasov equation, but $g$, the charge density, does not.

$$
\frac{\partial B}{\partial t}+\vec{f} \cdot[\vec{v}(\vec{x}, t) g]=0 .
$$

A point particle distribution,

$$
f(\vec{p}, \vec{x}, t)=\sum_{1} \delta\left[\vec{x}-\vec{x}_{1}(t)\right] \delta\left[\vec{p}-\vec{p}_{1}(t)\right] \text {, }
$$

does formally sat isfy the Vlasov equation.

We look for solutions of the fiteld equations which correspond more closely to "particles" than to a continuous fluid. Consider uncorrelated wave packets with radius $=0\left(r_{1}\right)$, where $r_{1}+0$ In the limit $h \rightarrow 0$. The wave number spread $\Delta k_{1}=0\left(1 / r_{1}\right)$, which gives a velocity dispersion $=\Delta k_{1} h=O\left(h ; r_{1}\right)$. To maintain a radius $=O\left(r_{1}\right)$ requires that $t=0\left(r_{1}{ }^{2}\right)$. The charge of the wave packet, $e_{1}$, is $o\left(r_{1}{ }^{3}\right)$, therefore self forces do not expand the wave facket to a radius greater than $O\left(r_{1}\right)$.

We define

$$
f(\vec{p}, \vec{x}, t)=\int d^{3} k_{1} w_{2}\left(\vec{k}_{1}-\vec{k}_{0}\right)\left[s_{1} g_{2}+c \cdot c \cdot\right] .
$$

where

$$
s_{1}=\int d^{3} x_{1} \exp \left[-1 \vec{k}_{1} \cdot \vec{x}_{1}\right] w_{1}\left(\vec{x}-\vec{x}_{1}\right) \phi\left(\vec{x}_{1}, t\right)
$$

and

$$
\begin{aligned}
& z_{2}=\int d^{3} x_{1} \exp \left[1 \vec{k}_{1} \cdot \vec{x}_{1}\right] w_{1}\left(\vec{x}-\vec{x}_{1}\right) \\
& {\left[-1 \partial_{t}-\alpha A_{0}\left(\vec{x}_{1}, t\right)\right] \phi^{*}\left(\vec{x}_{1}, t\right),}
\end{aligned}
$$

and

$$
\begin{aligned}
& \vec{k}_{0}=\vec{p} / \mathrm{a}+a \vec{A} . \\
& u_{1}\left(\vec{x}-\vec{x}_{1}\right)=\text { const, }\left|\vec{x}-\vec{x}_{1}\right|<r_{3},
\end{aligned}
$$$$
\text { - o. }\left|\vec{x}-\vec{x}_{1}\right|>r_{3}+r_{2} \text {. }
$$$$
H_{2}\left(\vec{k}_{-}-\vec{k}_{1}\right)=\operatorname{cons} t_{1}\left|\vec{k}_{k}-\vec{k}_{1}\right|<k_{3} \text {. }
$$

$$
=0, \quad\left|\vec{k}_{-}-\vec{k}_{1}\right|>k_{3}+k_{2} \text {. }
$$$$
\lim _{x \rightarrow 0} \frac{r_{1}}{r_{2}}, \frac{r_{2}}{r_{3}}, \frac{\Delta k_{1}}{k_{2}}, \frac{k_{2}}{k_{3}},
$$

$$
r_{3}, n k_{3}, n k_{3}^{2} r_{3}, r_{3}^{2} k_{3} \rightarrow 0
$$

$W_{1}$ and $w_{2}$ have continuous first order derivatives. We assume has only positive energy solutions. A more complicated defintition is necessary if there are also negative energy solutions. The distribution function is ensemble averaged because the number of wave packets in the support of $W_{1} \times W_{2}$ goes to zern in a single system. In the region where $d_{1} \times w_{2}$ is constant, the function acts as a ccunter of wave packets because it reduces (by Parseval's formula) to an integral over charge density. Only the asymptotic overle'p of these wave packets in the boundary region of $w_{1} \times w_{2}$ contribute to the derivatives of $f$. Derivatives of $\phi$ of $0(1)$ of these wave packets may then be neglected. since the volume of the boundary region over the cotal volume goes to zero, we may neglect derivatives of $\phi$ of $O(1)$ in general. This is loportant since derivatives of $O(1 / \mathrm{h})$ reflect the dynamics of charged particles, but derivatives of $O(1)$, which are necessary for charge conservation, are not directly connected with the particle dynamics. We need local solutions for $\phi$. Let

$$
\left.\Phi\left(\vec{x}_{1}, t_{1}\right)=\sum_{k} a_{k} \exp \left\{1 \mid \vec{k} \cdot \vec{x}_{1}-w\left(\vec{x}_{1}, t_{1}\right) t_{1}\right]\right\} .
$$


We assume that A changes slowly over distances of $O\left(r_{1}\right)$. Wavelengths of this size come from collisions between wave packets, and the colliston scrength vanishes in the 1imit. He expand $w$ in an asymptotic sertes in orders of $h$,

$$
\omega(\vec{x}, t)=\sum_{j=0}^{\infty} \omega_{j}(\vec{x}, t) .
$$

where $\omega_{j}=o\left(h^{j-1}\right)$,

and

$$
\omega_{j}(\vec{x}, c)=\sum_{n=0}^{\infty} w_{j n}(\vec{x}) t^{n} .
$$

Then $\omega$ may be solved expllclely. When only those terms necessary for the first order derivatives of $f(\vec{p}, \vec{x}, t)$ are kept, and $\omega$ is expanded about $(\vec{x}, c)$.

$$
\begin{gathered}
\qquad\left(\vec{x}_{1}, t_{1}\right)=\sum_{k} a_{k} \exp \left\{d \vec{k}=\vec{x}_{1}-(t,-t)\right. \\
\left.\left(\omega_{0}+\left(\operatorname{sv}^{\nu} A_{\mu a}\left(x_{1}-x\right)^{a}\right)\right\}\right\} .
\end{gathered}
$$

where $\omega_{0}, v$, and $A$ are defired at $(\vec{x}, e)$. (Double Greek fndexes indicate a sumation over all indexes; double lacin indexes Indicate a summation over space-11ke indexes.)

$$
\begin{aligned}
& p^{a}=h\left(k^{a}-a A^{a}\right) . \\
& p^{U}=\left(m^{2} c^{4}+c^{2} p^{a} p^{a}\right)^{1 / 2} . \\
& v^{\mu}=p^{\mu / p_{0}} . \\
& \omega_{0}=p_{0} m+a A_{0} .
\end{aligned}
$$

Application of the Viasov operator to $f$ gives

$$
\begin{aligned}
& {\left[v_{t}+v a_{a}+e(\vec{E}+\overrightarrow{v \times \vec{B}})^{a} \partial_{p} a\right] i} \\
& =\left(\partial_{t} f\right)_{k_{\theta}}+\left(v^{a} a_{i} f\right)_{k_{0}}-\sigma v^{\mu_{A}} \partial_{k_{0}} f .
\end{aligned}
$$

For $\mathrm{B}_{1}$, the three derivat lves in Ë. (29) give (inside the Fourler integral of $\diamond$ ), respectively,

$$
\text { (a) }-i\left[w_{v}(k)+a v^{\mu}(k) A_{u s}\left(x_{1}-x\right)^{a}\right]
$$

$$
\frac{-1\left[u_{b}\left(k_{0}\right)+\left(\frac{\partial \omega}{\partial k^{2}}\right)_{k_{0}}^{a}\left(k_{1}-k_{0}\right)^{a}+\left(\frac{\partial u}{\partial k}\right)_{k_{0}}^{a}\left(k-k_{1}\right)^{a}\right.}{\left.+a v^{\nu}(k) A_{\mu a}\left(x_{1}-x\right)^{a}\right]} .
$$

(b) $=i v^{a}\left(k_{1}-k\right)^{a}$.

and

$$
\begin{aligned}
& \text { (c) } \left.\operatorname{iav}^{\mu} \operatorname{Aus}^{x}\right)^{a}
\end{aligned}
$$

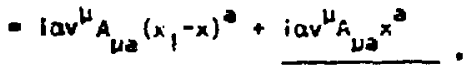

where

$$
\left(\frac{\partial \omega}{\partial k}\right)_{k_{0}}=v\left(k_{0}\right)^{a} \equiv v^{a}
$$

The undergatod terms are cancelled by the sase terms for $\mathrm{g}_{2}$. After cancellation we have

$$
\left.-\log _{\mu a}\left(x-x_{1}\right)^{a} \mid v\left(k_{0}\right) \cdots v(k)\right]^{\mu} \text {. }
$$

This cancels to the first approximetion, but the remainder is greater than $O(1)$. Fram $8_{2}$ we abtain the negative of term (31). The action of $\left(-1 \partial_{1}-\alpha A_{0}\right)$ on $\varphi^{*}$ produces a coef $f 1 c$ ient which multiplies each Fourier component of $\phi^{*}$. 


$$
\begin{aligned}
& \left\{\omega\left(k_{0}\right)-A_{u}(\vec{x}, c)\right\}+\left\{\omega(k)-s\left(k_{0}\right)\right. \\
& \left.-\alpha\left\{A_{0}\left(\vec{x}_{1},:\right)-A_{0}\left(\vec{x}_{0} t\right)\right]+\Delta v^{\mu_{A_{\mu}}}\left(x_{1}-x\right)^{*}\right\} .
\end{aligned}
$$

Term (j1) and its negative from $g_{2}$ cancel the first braces \{\} of cerm (32). This leaves terms of $o\left(r_{3}^{2} k_{3}\right), o\left(h k_{3}^{2} r_{3}\right) * o(1)$. The (logarithalc) de$r$ ivatiles of term (32) 1tself are of $O(1)$ and may be neglected. Then Eq. (29) is o(1) for $h \rightarrow 0$.

\section{REFERENCES}

1. D. Montgonery, "Stacistical Description of Plasmas" in Theory of the Unmagnetized Plasma (Gordon and Breach, New York, 1971), Chap. v111, Pp. 181-207.
2. W. Wylc and D. Pines; "Kinetic Equation for Plasma," Phys. Kev. 127, 1851 (1962).

3. D. DuBois, "Nonequilibrium Quantum Statistical Mechaniss of Plasmas and Padlation" in Leccures in Theoretical Physics (Gordon and Breach, New York, 1967, Vol, 9, pp. 489-620.

4. E. Harris, "Classical Plasma Phenomena from a Quantum Mechanical Ulewpoint," Adv. Plasma Phys. 3. 157 (1969).

5. I. Lerche, "Initlal-Value Prollem for Relativ1stic Plasma Oscillations," J. Math. Phys. $\underline{B}$, 1838 (196):

6. B. Foldert" $t$, "Thei.s $a$ iransverse waves in

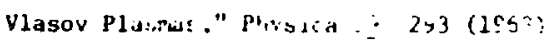

7. E. Weibel, "Sponcaneously Giulng Transverse Haves," Phys. Rev. Lett. 2, 83 (1959). 